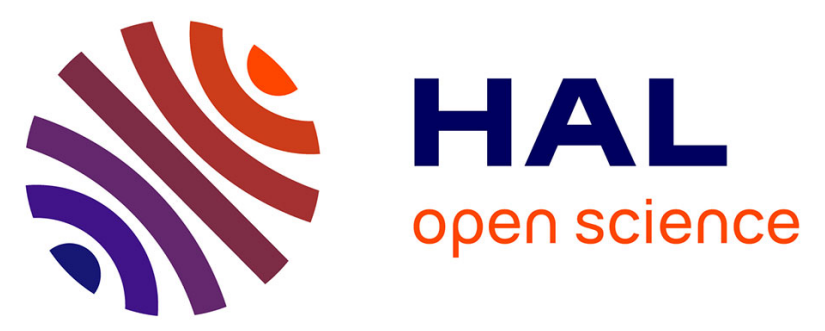

\title{
ZIF-8-basedvs.ZIF-8-derived Au and Pd nanoparticles as efficient catalysts for the Ullmann homocoupling reaction
}

W Wang, S Chen, Eduardo Guisasola Cal, M Moro, Sergio Moya, Emerson Coy, Changlong Wang, J.-R. Hamon, Didier Astruc

\section{To cite this version:}

W Wang, S Chen, Eduardo Guisasola Cal, M Moro, Sergio Moya, et al.. ZIF-8-basedvs.ZIF-8-derived $\mathrm{Au}$ and $\mathrm{Pd}$ nanoparticles as efficient catalysts for the Ullmann homocoupling reaction. Inorganic Chemistry Frontiers, 2020, 7 (20), pp.3945-3952. 10.1039/d0qi00831a . hal-02996428

\section{HAL Id: hal-02996428 \\ https://hal.science/hal-02996428}

Submitted on 17 Nov 2020

HAL is a multi-disciplinary open access archive for the deposit and dissemination of scientific research documents, whether they are published or not. The documents may come from teaching and research institutions in France or abroad, or from public or private research centers.
L'archive ouverte pluridisciplinaire HAL, est destinée au dépôt et à la diffusion de documents scientifiques de niveau recherche, publiés ou non, émanant des établissements d'enseignement et de recherche français ou étrangers, des laboratoires publics ou privés. 


\title{
ZIF-8-Based vs. ZIF-8-Derived Au and Pd Nanoparticles as Efficient Catalysts for the Ullmann Homocoupling Reaction
}

Wenjuan Wang, ${ }^{a, b}$ Shuang Chen, ${ }^{c}$ Eduardo Guisasola Cal, ${ }^{d}$ Marta Martínez Moro, ${ }^{d}$ Sergio Moya, ${ }^{d}$ Emerson Coy, ${ }^{e}$ Changlong Wang, ${ }^{a}$ Jean-René Hamon, ${ }^{b}$ and Didier Astruc ${ }^{\mathrm{a}, *}$

\footnotetext{
a. ISM, UMR CNRS 5255, Université de Bordeaux, Talence 33405 Cedex, France.

E-mail: didier.astruc@u-bordeaux.fr

b. Institut des Sciences Chimiques, UMR CNRS 6226, Université de Rennes 1, 35042

Rennes Cedex, France.

c. Department of Chemistry and Center for Atomic Engineering of Advanced

Materials, Anhui University, Hefei, Anhui 230601, China.

d. Soft Matter Nanotechnology Lab, CIC biomaGUNE, Paseo Miramón 182, 20014

Donostia-San Sebastián, Gipuzkoa, Spain.

e. NanoBioMedical Centre, Adam Mickiewicz University in Poznań, Wszechnicy

Piastowskiej 3, 61-614 Poznań, Poland.
}

\begin{abstract}
The Ullmann coupling reaction of aromatic halides is the most challenging C-C coupling reactions. Both MOF-based and MOF-derived types of catalysts have recently provided useful nanocatalysts for a variety of reactions, but they have rarely been compared. Here Ullmann-type aryl iodides homocoupling is efficiently catalyzed by gold and palladium nanoparticles (NPs) loaded on ZIF-8 before (ZIF-8-based nanocatalyst: M@ZIF-8) and after calcination (ZIF-8derived catalyst: M@CN). The nanocatalysts M@ZIF-8 (M = Pd, Au) are found to be more efficient than the catalysts M@CN. Pd@ZIF-8 shows better performances than those of Au@ZIF-8 for a series of aryl iodides, but in the case of 4-nitro-iodobenzene, deshydroiodination was observed besides the coupling product. Mechanisms are proposed and discussed for both $\mathrm{Au}(0)$ and $\mathrm{Pd}(0)$ nanocatalysts.
\end{abstract}

\section{Introduction}

The Ullmann reaction involving aryl halide coupling to biaryl has attracted attention for over a century, ${ }^{1}$ in particular given the synthetic interest of biaryl compounds, ${ }^{2-4}$ and the area has been the subject of excellent reviews. ${ }^{5-13}$ This reaction was well-known to be catalyzed by large amounts of copper catalyst, to require temperature higher than $200^{\circ} \mathrm{C}$ and to undergo extensive hydrodehalogenation as a side reaction. During the last decade, however, efforts have been devoted to circumvent direct aryl halide coupling and to use sophisticated Pd-based or Au-based catalysts. ${ }^{5-13}$ Au nanoparticles (NP) $)^{14-18}$ in particular were shown to be tentative catalysts. Jin's group has shown in several publications that small precisely molecularly defined $\mathrm{Au}$ nanoclusters covered with thiolate ligands were excellent catalysts of Ullmann coupling. ${ }^{15-18}$ Rodionov's group demonstrated that a combination of thiolate groups and linear semi-fluorinated chains effectively stabilized the NPs against aggregation, while preserving the catalytic activity of AuNPs. ${ }^{19}$ Various other reports using AuNP catalysts $^{20-24}$ have been recently and comprehensively reviewed by Ding's group, ${ }^{25}$ and particular attention to carboncarbon bond formation catalysts has been drawn by Garcia's group to the use of MOF-based catalysts. ${ }^{26,27} \mathrm{NP}$ catalysts are

Electronic Supplementary Information (ESI) available: UV-vis, XPS, ${ }^{1} \mathrm{H}$ NMR, TEM images and histograms, recycling results and catalytic reaction procedures. See DOI: $10.1039 / \times 0 \times x 00000 x$ indeed particularly efficient for a large variety of reactions and withstand homogeneous as well as heterogeneous reaction conditions. They present the great advantages of simple preparation by reduction of a metal salt, and do not require costly, sophisticated, and toxic ligands. ${ }^{28-37}$

MOF supports, particularly of ZIF-8 type have recently been particularly successful in the field of hydrogen generation and a number of other organic reactions. ${ }^{27,38,3929}$ MOFs are used in catalysis as templates that are all the more efficient as they act in synergy with NP catalysts (MOF-based catalysts). They also serve to form porous, very active $\mathrm{N}$-doped conducting carbon supports ("CN") upon pyrolysis at high temperature such as $900^{\circ} \mathrm{C}$, in which case they are classified as MOF-derived heterogeneous catalysts. Given the recent interest in both MOF-based and MOF-derived catalysts, ${ }^{40,41}$ here the efficiency and recyclability of ZIF-8-based and ZIF-8-derived catalysts in synergy with $\mathrm{Au}$ and Pd NPs for Ullmann homocoupling are compared. In this study ZIF-8-supported AuNPs and PdNPs are also compared, and optimization of the reaction conditions was conducted for the coupling of substituted aryl iodides.

\section{Results}

\section{Fabrication and characterization of the support and catalysts}

First, ZIF- $8^{42}$ was fabricated in methanol by an established method $^{43}$ (Supporting Information). Transmission electron microscopy (TEM) images (Fig. S1) display the morphology of ZIF-8 with hexagonal nanocrystals with a mean size of $92 \mathrm{~nm}$. Based on the deposition-precipitation (DP) method, rapid reduction by $\mathrm{NaBH}_{4}$ of metal cations in $\mathrm{HAuCl}_{4}$ or $\mathrm{NaPdCl}_{4}$ in a ZIF-8 suspension was executed to afford metal NP loading in ZIF-8 (M@ZIF-8). Then M@ZIF-8 was collected by centrifugation, successively washed with $\mathrm{H}_{2} \mathrm{O}$ and dried in vacuo overnight. Then M@ZIF- 8 was pyrolyzed at $900^{\circ} \mathrm{C}$ for $2 \mathrm{~h}$ 
under $\mathrm{N}_{2}$ to form the MOF-derived metal nanoparticles M@CN as shown in Scheme 1.
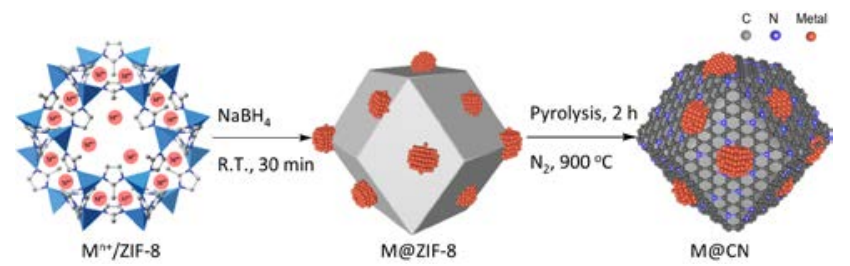

Scheme 1. Synthesis of the M@ZIF-8 and M@CN (M=Au, Pd).
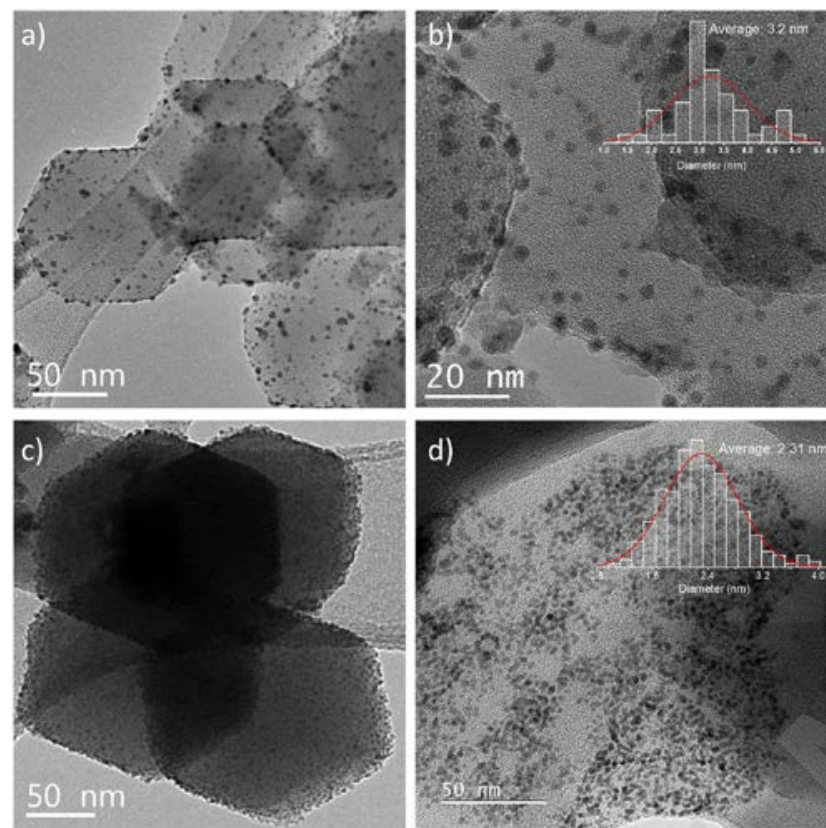

Fig. 2 TEM images of Au@ZIF-8; a and b (the insert is the size distribution histogram of Au@ZIF-8) and Pd@ZIF-8 (c).d) TEM image and size distribution of Pd@ZIF-8 after digestion of the nanocatalyst with ethylenediamine tetracetic acid (EDTA) to release nanoparticles, and nanoparticles stabilized by PVP.

According to the TEM of the as-prepared Au@ZIF-8, the AuNPs are homogeneously loaded on hexagonal ZIF-8 (Fig. 2a) with a mean size of 3.4 nm (Fig. 2b insert). In the TEM of Pd@ZIF-8 (Fig.2c), it is not easy to observe the PdNPs on the ZIF-8 support. Therefore, a common method, digestion of the nanocatalyst with EDTA to release nanoparticles that are further stabilized by PVP, was used to obtain the average size of Pd@ZIF-8: $2.31 \mathrm{~nm}$ as shown in Fig. 2d insert, which is smaller than Au@ZIF-8.

Compared with ZIF-8, no peak of either Pd NP or Au NP species was observed in the X-ray powder diffraction (PXRD) patterns after reduction (Fig. S5), which implies that both Pd@ZIF-8 and Au@ZIF-8 have a too low Au loading or an ultra-small size. ${ }^{44,45}$

$X$-ray photoelectron spectroscopy (XPS) was employed to check the metal oxidation state and composition. XPS results revealed the presence of $\mathrm{Zn}, \mathrm{Pd}, \mathrm{O}, \mathrm{C}$ and $\mathrm{N}$ in the catalyst Pd@ZIF-8 (Fig. S6a). As shown in Fig. S6b, the well-defined peaks at 335.2 and $340.7 \mathrm{eV}$ corresponding to $\mathrm{Pd}(0) 3 \mathrm{~d}_{5 / 2}$ and $\operatorname{Pd}(0) 3 d_{3 / 2}$, and two peaks at 338.1 and $343.7 \mathrm{eV}$ are also observed for $\operatorname{Pd}(I I) 2 p_{5 / 2}$ and $\operatorname{Pd}(I I) 2 p_{3 / 2}$ respectively. For
Au@ZIF-8, similar elements, Zn, O, C and N are observed from Fig. S6c. The peaks at 83.6 and $87.3 \mathrm{eV}$ belong to $\mathrm{Au}(0) 4 \mathrm{f}_{7 / 2}$ and $\mathrm{Au}(0) 4 \mathrm{f}_{5 / 2}$, and two peaks at 88.7 and $90.8 \mathrm{eV}$ are also observed for $\mathrm{Zn}$ (II) $2 \mathrm{p}_{3 / 2}$ and $\mathrm{Zn}$ (II) $2 \mathrm{p}_{1 / 2}$ respectively (Fig. S6d).

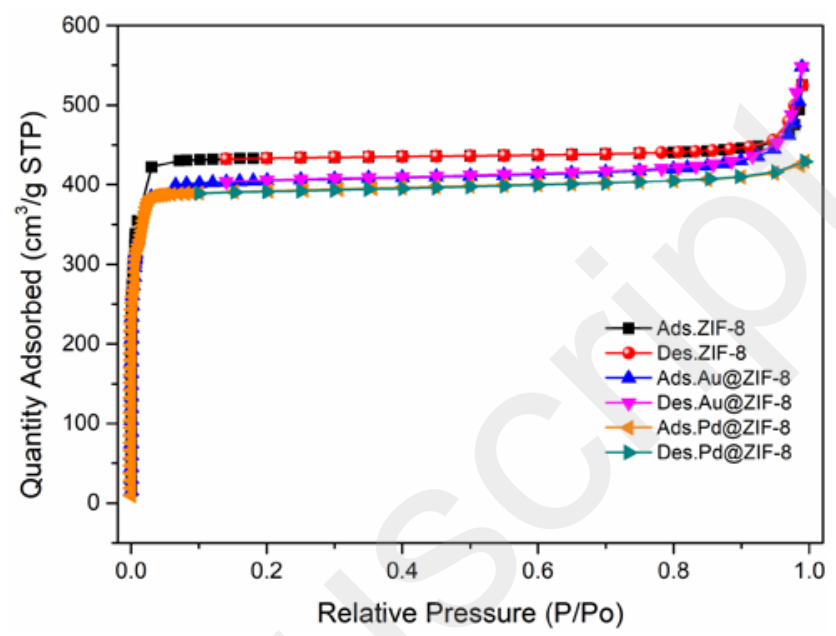

Fig. 2 Nitrogen adsorption-desorption isotherms of ZIF-8, Au@ZIF-8 and Pd@ZIF-8.

Table 1. lodobenzene coupling catalyzed by various catalysts.

\begin{tabular}{|c|c|c|c|c|}
\hline Entry & Catalyst & $\begin{array}{c}\text { Metal loading } \\
\text { (wt \%) }\end{array}$ & $\begin{array}{l}\text { Metal\% } \\
\text { (mmol \%) }\end{array}$ & $\begin{array}{c}\text { Conversion } \\
(\%)^{b}\end{array}$ \\
\hline 1 & Au@ZIF-8 & 5 & 6 & 76 \\
\hline 2 & Au@ZIF-8 & 10 & 12 & 83 \\
\hline 3 & Pd@ZIF-8 & 2.7 & 6 & 86 \\
\hline 4 & Pd@ZIF-8 & 5.4 & 12 & 89 \\
\hline 5 & Pd@ZIF-8 & 1.35 & 3 & 25 \\
\hline 6 & ZIF-8 & 0 & 0 & 0 \\
\hline 7 & $\mathrm{Au} @ \mathrm{CN}^{\mathrm{a}}$ & 13 & 6.5 & 40 \\
\hline 8 & $\mathrm{Pd} @ \mathrm{CN}^{\mathrm{a}}$ & 7 & 6.5 & 13 \\
\hline 9 & $\mathrm{CN}^{\mathrm{a}}$ & 0 & 0 & 0 \\
\hline \multicolumn{5}{|c|}{ Catalyst, $\mathrm{K}_{2} \mathrm{CO}_{3}$} \\
\hline
\end{tabular}

Reaction conditions: $0.2 \mathrm{mmol}$ iodobenzene, $0.6 \mathrm{mmol} \mathrm{K}_{2} \mathrm{CO}_{3}, 1 \mathrm{~mL} \mathrm{DMF}, 50 \mathrm{mg}$ M@ZIF-8, $130{ }^{\circ} \mathrm{C}, \mathrm{N}_{2} .{ }^{\mathrm{a}} \mathrm{M} @ Z I F-8$ pyrolyzed at $900{ }^{\circ} \mathrm{C}$ for $2 \mathrm{~h}$ under $\mathrm{N}_{2}, 20 \mathrm{mg} \mathrm{M@CN.}{ }^{b}$ ${ }^{1} \mathrm{H}$ NMR conversion.

$\mathrm{N}_{2}$ physisorption measurements of Brunauer-Emmett-Teller (BET) surface areas for ZIF-8, Pd@ZIF-8 and Au@ZIF-8 were determined to be $1365.1,1247.7$ and $1283.1 \mathrm{~m}^{2} \mathrm{~g}^{-1}$, respectively (Fig. 2 and Table S1). This appreciable decrease means that the pores of ZIF-8 are embedded with MNPs or/and covered by MNPs loading on the surface.

\section{Catalytic activity}


In order to optimize the reaction conditions, iodobenzene was first chosen as the model substrate for the classical Ullmann C-C homocoupling reaction, Au@ZIF-8 (with various Au loadings) as catalyst, $\mathrm{K}_{2} \mathrm{CO}_{3}$ as a base and dimethyl formamide (DMF) as solvent at $130^{\circ} \mathrm{C}$ under $\mathrm{N}_{2}$ atmosphere for 2 days. behavior analogous to Au@ZIF-8 with twice more metal loading (Table 1, entries 3 ). In addition, the nanocatalyst Pd@ZIF-8 with 1.35 and 5.4 wt\% of Pd loading (Table 1, entries $4,5)$ was used to optimize the metal loading rate. The ${ }^{1} \mathrm{H}$ NMR results indicate that $2.7 \mathrm{wt} \%$ of $\mathrm{Pd}$ is good enough to get an excellent conversion to iodobenzene.

Then different catalysts were applied in the above model reaction, and the MOF-based catalysts Au@ZIF-8 and Pd@ZIF8 with the same metal loading showed an excellent catalytic performance under the same conditions. Interestingly, the catalytic activity of MOF-derived catalysts with larger sizes considerably decreased (Table 1 , entries 7,8 ) after calcination of M@ZIF-8 at $900{ }^{\circ} \mathrm{C}$ under $\mathrm{N}_{2}$, which may be due to the aggregation of MNPs. In control experiments, no reaction occurred using the support ZIF-8 or $\mathrm{CN}$ as the only catalyst (Table 1, entries 6,9). In light of the above results, Au@ZIF-8 and Pd@ZIF-8 were chosen as catalysts to further investigate and optimize catalytic performances and screen out the reaction conditions. With Au@ZIF-8 the investigation of the optimal reaction temperature in this Ullmann homocoupling reaction included conducting the model reaction at $110^{\circ} \mathrm{C}$ and $140^{\circ} \mathrm{C}$ (Table S2, entry 2-3), and it was found that Au@ZIF-8 worked best at $140^{\circ} \mathrm{C}$. Two other solvents with high boiling point (xylene and PEG-400) were probed for the reaction (Table S2, entries 4-5), but they were not as satisfactory as DMF (Table S2, entry 3). In particular, the reactions using toluene produced poor yields. Indeed, the reaction stoichiometry required the consumption of two electrons, and DMF and alcohols are good electron donors at such high temperature, whereas toluene is not. Thus, DMF that contributes to the stabilization of the NPs also acts as a reductant. Among the different bases that were probed in this system including $\mathrm{K}_{2} \mathrm{CO}_{3}$ and $\mathrm{K}_{3} \mathrm{PO}_{4}$ (Table $\mathrm{S} 2$, entries 3, 6), the catalyst Au@ZIF-8 showed the best conversion efficiency (83\%) with $\mathrm{K}_{3} \mathrm{PO}_{4}$. Analogous studies were also conducted with
According to the ${ }^{1} \mathrm{H}$ NMR results, the conversion of iodobenzene increased slightly from $76 \%$ to $83 \%$ with the amount of Au changing from $6 \mathrm{mmol} \%$ to $12 \mathrm{mmol} \%$ (Table 1, entries 1-2). Under the same conditions, Pd@ZIF-8 even with 6 mmol\% of Pd exhibits a

Table 2. Effects of base and temperature on the homocoupling of iodobenzene catalyzed by Pd@ZIF-8.

\begin{tabular}{ccccc} 
& \multicolumn{4}{c}{ Pd@ZIF-8, base } \\
\cline { 3 - 5 } BMF, Temp. ${ }^{\mathbf{0}} \mathbf{C}$, Time & Time & Temp. & Conversion (\%) ${ }^{\mathrm{a}}$ \\
\hline 1 & $\mathrm{~K}_{2} \mathrm{CO}_{3}$ & 24 & 130 & 33 \\
3 & $\mathrm{~K}_{2} \mathrm{CO}_{3}$ & 48 & 130 & 86 \\
4 & $\mathrm{~K}_{3} \mathrm{PO}_{4}$ & 48 & 130 & 92 \\
\hline $\mathrm{K}_{3} \mathrm{PO}_{4}$ & 48 & 140 & 100
\end{tabular}

Reaction conditions: $0.2 \mathrm{mmol}$ iodobenzene, $0.6 \mathrm{mmol}$ base, $1 \mathrm{~mL}$ solvent, $50 \mathrm{mg}$ Pd@ZIF-8 (6 mmol\% Pd), $\mathrm{N}_{2} .{ }^{\text {a }} \mathrm{H}$ NMR conversion.

Pd@ZIF-8. It was shown that Pd@ZIF-8 best cooperated with $\mathrm{K}_{3} \mathrm{PO}_{4}$ to catalyze the model reaction at $140^{\circ} \mathrm{C}$ for 2 days (Table 2, entries 1-4).

Finally, the scope of the substrates was extended under these optimized reaction conditions. As shown in table 3, $100 \%$ of $4-$ iodo-toluene, $100 \%$ of 4 -iodo-anisole and $67 \%$ of 4 -chloroiodobenzene are consumed to form the final product $4,4^{\prime}$ dimethoxy-1,1'-biphenyl, 4,4'-dimethyl-1,1'-biphenyl and 4,4'dichloro-1,1'-biphenyl (Table 3). With Au@ZIF-8 as the catalyst, however, in comparison with the conversions above, a lower yield for each electron-rich substrate than with Pd@ZIF-8 was obtained (Table 3), which further demonstrated that Pd@ZIF-8 was the best catalyst. For the electron-deficient substrates, however, Au@ZIF-8 and Pd@ZIF-8 show a similar performance. The case of 1-iodo-4-nitrobenzene was found to be special. The substrate was totally consumed at $140^{\circ} \mathrm{C}$, but the yield of coupled product was only $50 \%$ with $50 \%$ of the side product

Table 3. Homocoupling of aryl iodides by Pd@ZIF-8 (Au@ZIF-8).

$\frac{\text { Substrate }}{\text { Conversion (\%) }}$



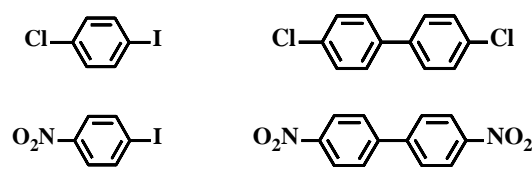

$100\left(47^{b}+53^{c}\right) \quad 100\left(50^{b}+50^{c}\right)$

Reaction conditions: $0.2 \mathrm{mmol}$ substrate, $0.6 \mathrm{mmol} \mathrm{K}_{3} \mathrm{PO}_{4}, 1 \mathrm{~mL}$ DMF, $50 \mathrm{mg}$ M@ZIF-8 (6 mmol\% metal), $140{ }^{\circ} \mathrm{C}, \mathrm{N}_{2}, 48 \mathrm{~h} .{ }^{a}{ }^{1} \mathrm{H}$ NMR conversion. ${ }^{b}$ Yield of C-C homocoupling. ${ }^{\mathrm{C}}$ Yield of dehydrohalogenation.

Table 4. Sizes and catalytic efficiencies of the nanocatalysts ${ }^{a}$

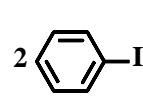

Catalyst, $\mathrm{K}_{3} \mathrm{PO}_{4}$

DMF, $140{ }^{\circ} \mathrm{C}, 48 \mathrm{~h}$

\begin{tabular}{|c|c|c|c|c|}
\hline Catalyst & $\begin{array}{c}\text { Size }^{b} \\
(\mathbf{n m})\end{array}$ & $\begin{array}{c}\text { Size }^{c} \\
(\mathbf{n m})\end{array}$ & $\begin{array}{c}\text { Conversion } \\
\mathbf{( \% )}^{\boldsymbol{d}}\end{array}$ & $\begin{array}{r}\text { Conversion-1 }^{\text {st }} \\
\text { Cycle (\%) }^{\boldsymbol{e}}\end{array}$ \\
\hline $\mathrm{Au} @ \mathrm{ZIF}-8$ & 3.20 & 23.14 & 83 & 54 \\
$\mathrm{Au} @ \mathrm{CN}^{f}$ & 4.39 & $/$ & 40 & 20 \\
$\mathrm{Pd} @ \mathrm{ZIF}-8$ & 2.31 & 3.16 & 100 & 75 \\
${\mathrm{Pd} @ \mathrm{CN}^{f}}^{5}$ & 5.64 & $/$ & 13 & 9 \\
\hline
\end{tabular}

${ }^{a}$ Reaction conditions: $0.2 \mathrm{mmol}$ iodobenzene, $0.6 \mathrm{mmol} \mathrm{K}_{3} \mathrm{PO}_{4}, 1 \mathrm{~mL} \mathrm{DMF}, 50 \mathrm{mg}$ M@ZIF-8 (6 mmol\% metal), $140^{\circ} \mathrm{C}, \mathrm{N}_{2}, 48 \mathrm{~h}$. TEM size before reaction (size ${ }^{b}$ ) and after reaction $\left(\right.$ size $\left.e^{c}\right) .{ }^{d 1} \mathrm{H}$ NMR conversion. ${ }^{e}$ Conversion after the 1 rst cycle. ${ }^{f} 20 \mathrm{mg} \mathrm{M@CN}$ (6.5 mmol\% metal).

nitrobenzene resulting from dehydrohalogenation that was not found with the other $p$-substituted iodoarenes examined. This result is similar to the case of Au@ZIF-8.

\section{Recyclability of Au@ZIF-8 and Pd@ZIF-8}

The conversion of iodobenzene decreased from $100 \%$ to $75 \%$ after the recycling of Pd@ZIF-8 for the model reaction, which can probably be ascribed to the loss of some Pd species (Fig. S7a) It was found by TEM, however, that after the reaction PdNPs became larger (Table 4 and Fig.S4). For Au@ZIF-8, the conversion decreased a lot from $83 \%$ to be $43 \%$ after the 1 st cycle, which is due to the size of AuNPs with a remarkable increase and serious aggregation (Table 4 and Fig.S5). Besides, the signal of $A u(0)$ of Au@ZIF-8 decreased in the XPS spectrum (Fig. S7b). Moreover, both Au@ ZIF-8 and Pd@ZIF-8 suffer from leaching as observed by the yellow color of the solution after the reaction. For the ZIF-8 derived nanocatalyst, the recycling yield is very low, 20\% for Au@CN and 9\% for Pd@CN (Table 4).

\section{Discussion}

Mechanistic discussions on Ullmann coupling catalyzed by inorganic complexes have been reviewed by de Vries and van Koten. ${ }^{9}$ Here the specificity of the metal (0) NP catalysts is discussed. The rather high temperature needed for the Ullmann reaction results from the absence of transmetalation step $^{46}$ that renders $\mathrm{C}-\mathrm{C}$ coupling facile in other cross coupling reaction of aryl halides with organoboron and other organometallic reagents. The difficulty in Ullmann coupling is the double oxidative addition of the aryl halide that requires the intermediacy of high oxidation states with monometallic catalysts. ${ }^{47}$ Another crucial problem is that this oxidative addition requires the presence of classic reductant of the metal intermediate species to the starting zero oxidation state. ${ }^{48}$ With PdNPs, it is well known that the leaching mechanism observed here is most often encountered in carbon-carbon coupling reaction, even when they are much more facile. ${ }^{28}$ Here the high temperature needed for the reaction renders the integrity of the NP doubtful, and the considerable structural change of the NP sizes along the reaction as well as the observed leaching of the PdNPs show that atoms and small clusters of atoms are formed along the reaction pathways.

Thus, it is difficult to claim the actual NP or cluster size during the catalytic events. Under the reaction conditions, the surface Au and $\mathrm{Pd}$ atoms are shown by XPS to be in the zero-oxidation state, which is expected given the full reduction of the cationic precursors and the $L$ (2-electron)-ligand nature of the DMF ligands. It is well known that oxidative addition of aryl iodides is easy on $\operatorname{Pd}(0)$ to start the catalytic cycles, and that the Pd oxidation states involved are 0 and II, i.e. that inner-sphere two-electron transfers are involved in the catalytic steps. Such is not the case for the starting $\mathrm{Au}(0)$ that can only undergo single- electron transfer to $\mathrm{Au}(\mathrm{I})$, eventually before further oxidative addition to $\mathrm{Au}(\mathrm{III})$. Thus, we suggest that the AuNP first acts as reductant of $\mathrm{Ar}-\mathrm{X}$ in the initial step. Such a step is endergonic, but it becomes possible at high temperature, singleelectron transfers being favored at high temperature, especially given the irreversibility of the follow-up step. The redox step generates $\mathrm{X}^{-}, \mathrm{Ar}$ and $\left[\mathrm{Au}_{n}\right]^{+}$, and it is followed by cage coupling between the above radical cage followed by hydrogen atom abstraction from the solvent. Indeed the life time of the nitrophenyl radical is considerably prolonged compared to the phenyl radical and other aryl radicals due to the pelectronic delocalization provided by the nitro group. This observation of the dehydrohalogenation in this particular case somewhat confirms the proposal of aryl iodide reduction producing 


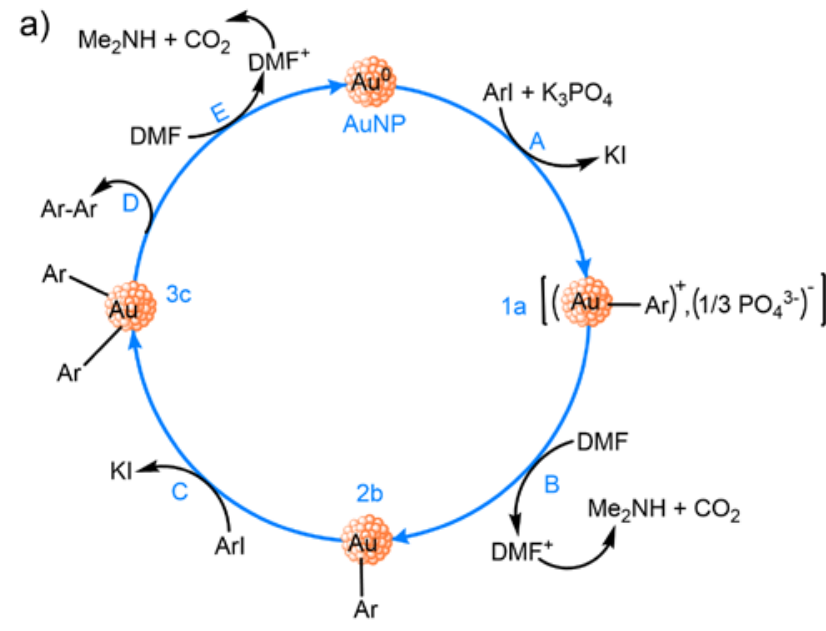

b)

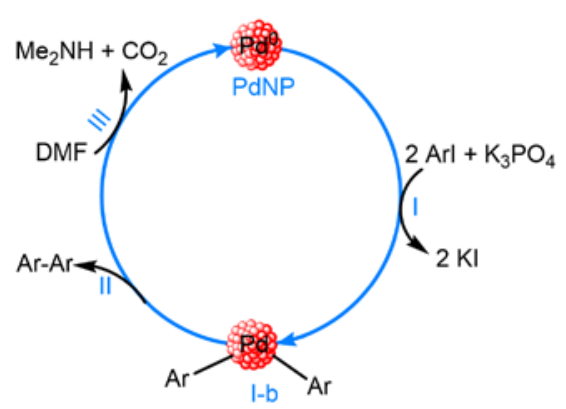

Scheme 2. Proposed catalytic cycles for the Ullmann homocoupling reaction catalyzed by Au@ZIF-8(a) and Pd@ZIF-8(b). The small colored spheres represent a NP, not a single atom (except if single leached atoms are involved). Coordination of ligands in the catalytic cycle may involve several surface atoms of the NP or cluster, and the aryl ligands may "walk" on the surfaces towards gathering for reductive elimination.

the radical cage in this first step. The overall stoichiometry of the Ullmann coupling requires two electrons (equation 1), which means that the only possible stoichiometric electron donor is the solvent (DMF and alcohols).

$$
2 \mathrm{Arl}+2 \mathrm{e}^{-} \rightarrow \mathrm{Ar}-\mathrm{Ar}+2 \mathrm{I}^{-}
$$

(equation 1)

The solvents DMF (that is oxidized in situ to $\mathrm{Me}_{2} \mathrm{NH}$ and $\left.\mathrm{CO}_{2}\right)^{49}$ and PEG are known to be good electron donors, and they are indeed presently suitable and favorable contrary to toluene. Note that DMF plays multiple roles as solvent, stabilizing 2electron $L$ ligand and reducing agent. DMF has even been used to serve as reducing agent of transition metal cations for the formation of NPs, in particular $\mathrm{Ag}^{+}$to $\mathrm{Ag}^{0},{ }^{49}$ which shows its ability to serve in single-electron transfer steps.

We suggest that with PdNPs this reducing function is used in the last step of the catalytic cycle (most probably decomposed in two successive single-electron transfer steps) and in the steps involving reduction of cationic AuNP to neutral species in the cycle with AuNP. These endergonic redox steps of the cycles are most probably the rate-limiting steps of the overall reactions and responsible for the high temperatures required for these Ullmann coupling with both the AuNP and PdNP catalysts. It is interesting that dehydroiodination of 4-nitroiodobenzene also intervenes in the PdNP-catalyzed reaction, as in the case of AuNP, although to a lower extent. This indicates that reduction of this easily reducible substrate (because of the strongly electron withdrawing nitro group) occurs in competition with oxidative addition onto $\mathrm{Pd}(0)$ at this high reaction temperature. The optimization of the base used in the reactions ended up in the finding that $\mathrm{K}_{3} \mathrm{PO}_{4}$ was the best base in terms of performance. In fact, the so-called "base" does not function as a base but as a salt in which the electrostatic bonding between the anion and the cation plays the key role in the iodide removal from the metal creating new ion pairs as shown in scheme 2 . Thus, it is the relative ion pairing strengths among the distinct ion pairs involved in the catalytic cycle that play the decisive role in the rate of the reaction. ${ }^{50,51}$

The comparison between ZIF-8-based catalysts and ZIF-8derived that had to our knowledge not been recorded earlier provides, under the same conditions, yields that are almost quantitative with ZIF-8 based catalysts and significantly or sharply lower with ZIF-8-derived catalysts. It is possible that the confinement in ZIF-8-based catalysts provides a better stability and efficiency of the NPs than the ZIF-8 derived catalyst for the Ullmann reaction that requires harsh conditions. Therefore, the ZIF-8-based and ZIF-8-derived nanocatalysts have been examined for both $\mathrm{Au}$ and $\mathrm{Pd}$.

In comparison with Pd@CN, Pd@ZIF-8 shows a much better performance in the same conditions, but with Pd@ZIF-8 leaching prevents efficient recycling. However, both Au@ZIF-8 and Au@CN display a similar conversion.

\section{Experimental}

\section{General data}

\section{Preparation of ZIF-8. ${ }^{42}$}

2-Methylimidazole (75 mmol, $6.16 \mathrm{~g}$ ) was dissolved in $150 \mathrm{~mL}$ methanol at room temperature (R.T.) forming a clear solution in flask A. $\mathrm{Zn}\left(\mathrm{NO}_{3}\right)_{2} \cdot 6 \mathrm{H}_{2} \mathrm{O}(20 \mathrm{mmol}, 6.02 \mathrm{~g})$ was dissolved in $150 \mathrm{~mL}$ methanol in the flask $B$. Then the solution in the flask $B$ was added into flask A. After 1 day, the white suspension was collected by centrifugation and washed by methanol three times. ZIF-8 was dried at $60^{\circ} \mathrm{C}$ overnight.

\section{Preparation of the nanocatalysts M@ZIF-8.}

$100 \mathrm{mg}$ of ZIF- 8 was dispersed in $5 \mathrm{~mL}$ methanol under ultrasound for $5 \mathrm{~min}$, then $1 \mathrm{~mL}$ aqueous solution with $5 \mathrm{mg}$ metal $\left(\mathrm{HAuCl}_{4} \cdot 3 \mathrm{H}_{2} \mathrm{O}\right.$ or $\left.\mathrm{Na}_{2} \mathrm{PdCl}_{4}\right)$ and $4 \mathrm{~mL}$ deionized water were added respectively, and the mixture was submitted to ultrasound for another $5 \mathrm{~min}$. Then stirring was continued for $30 \mathrm{~min}$ at room temperature (rt). Then, $1 \mathrm{~mL}$ aqueous solution of fresh $\mathrm{NaBH}_{4}(10$ equiv. per metal) was rapidly injected under vigorous stirring. After $30 \mathrm{~min}$, the final product was collected by centrifugation at 10000 r.p.m. for $10 \mathrm{~min}$. and washed three times with deionized water, then dried at $60^{\circ} \mathrm{C}$ in vacuo overnight. The metal content was quantified by inductively coupled plasma atomic emission 
spectroscopy (ICP-AES). The ICP result showed that there was 4.5 wt \% Au in Au@ZIF-8 and 2.3 wt\% Pd in Pd@ZIF-8.

\section{Preparation of Au@CN and Pd@CN.}

The powder of Au@ZIF-8 (or Pd@ZIF-8) sample was placed in a tube furnace, then heated to $900^{\circ} \mathrm{C}$ for $2 \mathrm{~h}$ with a heating rate of $5^{\circ} \mathrm{C}$ $\mathrm{min}^{-1}$ under a flow of $\mathrm{N}_{2}$ before cooling to rt providing Au@CN (or Pd@CN).

\section{Application in catalysis}

A $10-\mathrm{mL}$ Schlenk tube was charged with a magnetic bar, $50 \mathrm{mg}$ M@ZIF-8 or 20 mg M@CN, $0.6 \mathrm{mmol} \mathrm{K}_{3} \mathrm{PO}_{4}, 0.2 \mathrm{mmol}$ aryl iodides and $1 \mathrm{~mL}$ DMF and purged with $\mathrm{N}_{2}$ for 10 minutes. Then the mixture was heated at $140^{\circ} \mathrm{C}$ for $48 \mathrm{~h}$, and after cooling down to rt, the reaction mixture was filtered. The liquid was extracted several times with dichloromethane and water in order to remove DMF. The organic phase was dried over $\mathrm{Na}_{2} \mathrm{SO}_{4}$, then $\mathrm{CH}_{2} \mathrm{Cl}_{2}$ was removed under reduced pressure at the rotary evaporator providing the crude product. Finally, the result of the homocoupling Ullmann reaction was monitored via ${ }^{1} \mathrm{H} \mathrm{NMR}\left(300 \mathrm{MHz}, \mathrm{CDCl}_{3}\right)$.

\section{Conclusion}

The carbon-carbon homocoupling of various aryl iodides is efficiently catalyzed by MOF-based metal NPs and MOFderived metal NPs, but the MOF-based nanocatalysts perform better than the related MOF-derived catalysts (MOF = ZIF-8). Both PdNPs and AuNPs are efficient, although the ZIF-8-based PdNPs are slightly better than their nanogold analogues. With these results in hand, mechanisms are proposed for both the $\operatorname{Pd}(0)$ and $\mathrm{Au}(0)$ nanocatalysts. Since the stoichiometry of the Ullmann reaction requires two electrons, these electrons are provided by the DMF and PEG-400 that are good reductants, whereas poor reductants such as toluene give bad coupling yields. The difference between the catalytic cycles of the $\mathrm{Pd}(0)$-catalyzed and $\mathrm{Au}(0)$-catalyzed reactions must take into account the fact that $\mathrm{Pd}(0)$ readily give rise to oxidative addition with increase of two units of the metal oxidation state, whereas $\mathrm{Au}(0)$ does not. Thus, double oxidative addition occurs at the beginning of the cycle with $\operatorname{Pd}(0)$, whereas the $\mathrm{Au}(0)$ cycle must start by reduction of the aryl iodide providing an $\mathrm{Au}(\mathrm{I})$-aryl bond. The double oxidative addition on $\mathrm{Pd}(0)$ probably does not reach Pd(IV), but may more easily and successively occur on two distinct $\operatorname{Pd}(0)$ atoms of the PdNP surface to give rise to two $\mathrm{Pd}(\mathrm{II})$-aryl species from which arylaryl reductive elimination occurs (eventually following some "walk" of the aryl ligands on the Pd surface until reaching each other to reductively eliminate). In both the $\mathrm{Pd}$ and $\mathrm{Au}$ catalytic cycles, the two electrons are transferred stepwise (see Scheme 2). Finally, these results with NPs may be compared with the remarkable results obtained by Jin's group with $\mathrm{Au}_{25}$ nanoclusters that were obtained under slightly milder conditions. One major difference is that with the $\mathrm{Au}_{25}$ nanoclusters, the sulfide-bonded $\mathrm{Au}$ atoms are in the oxidation state $A u(I)$, thus in principle the difficult $A u(0) \rightarrow(A u(I)$ step of
Scheme 2 is not required in the case of the nanocluster catalysts. This may represent one of the significant advantages of the use of the $\mathrm{Au}_{25}$ nanoclusters. Note that a similar situation was reached by Rodionov et al in their use of thiolate-stabilized AuNPs. In conclusion the excellent coupling results and comparison between ZIF-8 based and ZIF-8 derived templates of AuNPs and PdNPs and the comparison between the AuNP and PdNP catalysts together with the scrutiny of the optimized temperature, base and solvent allow a better understanding of the optimization and mechanisms of the Ullmann coupling reactions and their potential applications.

\section{Conflicts of interest}

The authors declare no competing financial interest.

\section{Acknowledgements}

Financial support from the China Scholarship Council of the People's Republic of China (PhD grant to W. W.), the University of Bordeaux, the Centre National de la Recherche Scientifique (CNRS) and CIC biomaGUNE is gratefully acknowledged.

\section{Notes and references}

1 F. Ullmann and J. Bielecki, Ueber synthesen in der biphenylreihe, Ber. Dtsch. Chem. Ges., 1901, 34, 2174.

2 G. Evano, N. Blanchard and M. Toumi, Copper-mediated coupling reactions and their applications in natural products and designed biomolecules synthesis, Chem. Rev., 2008, 108, 3054.

3 G. Bringmann, T. Gulder, T.A. Gulder and M. Breuning, Atroposelective total synthesis of axially chiral biaryl natural products, Chem. Rev., 2011, 111, 563.

4 H. Aldemir, R. Richarz and T.A. Gulder, The biocatalytic repertoire of natural biaryl formation, Angew. Chem. Int. Ed., 2014, 53, 8286

5 J. Hassan, M. Sévignon, C. Gozzi, E. Schulz and M. Lemaire, Aryl-aryl bond formation one century after the discovery of the Ullmann reaction, Chem. Rev. 2002, 102, 1359.

6 I. P. Beletskaya and A. V. Cheprakov, Copper in crosscoupling reactions: the post-Ullmann chemistry, Coord. Chem. Rev., 2004, 248, 2337.

7 J.-P. Corbet and G. Mignani, Selected patented crosscoupling reaction technologies, Chem. Rev., 2006, 106, 2651.

8 D. Alberico, M.E. Scott and M. Lautens, Aryl-aryl bond formation by transition-metal-catalyzed direct arylation, Chem. Rev., 2007, 107, 174.

9 E. Sperotto, G. P. M. van Klink, G. van Koten and J. G. de Vries, The mechanism of the modified Ullmann reaction, Dalton Trans., 2010, 39, 10338.

10 C. Sambiagio, S. P. Marsden, A. J. Blacker and P. C. McGowan, Copper catalysed Ullmann type chemistry: from mechanistic aspects to modern development, Chem. Soc. Rev., 2014, 43, 3525.

11 Q.-T. Fan, J. M. Gottfried and J.-F. Zhu, Surface-catalyzed C$C$ covalent coupling strategies toward the synthesis of lowdimensional carbon-based nanostructures, Acc. Chem. Rev., 2015, 48, 2484.

$12 \mathrm{~S}$. Mondal, Recent advancement of Ullmann-type coupling reactions in the formation of $\mathrm{C}-\mathrm{C}$ bond, ChemTexts, 2016, 2, 17. 
13 Y. Zhou and G. Li. A Critical review on carbon-carbon coupling over ultra-small gold nanoclusters, Acta Phys.-Chim. Sinica, 2017, 33, 1297.

14 M-C. Daniel and D. Astruc, Gold nanoparticles: assembly, supramolecular chemistry, quantum-size-related properties, and applications toward biology, catalysis, and nanotechnology, Chem. Rev., 2004, 104, 293.

15 G. Li and R.-C. Jin, Catalysis by gold nanoparticles: carboncarbon coupling reactions, Nanotechnol. Rev., 2013, 2, 529.

$16 \mathrm{G}$. Li, C. Liu, Y. Lei and R.-C. Jin, $\mathrm{Au}_{25}$ nanocluster-catalyzed Ullmann-type homocoupling reaction of aryl iodides, Chem. Commun., 2012, 48, 12005.

17 G. Li, H. Abroshan, C. Liu, S. Zhuo, Z.-M. Li, Y. Xie, H. J. Kim, N L. Rosi and R.-C. Jin, Tailoring the electronic and catalytic properties of $\mathrm{Au}_{25}$ nanoclusters via ligand engineering, ACS Nano, 2016, 10, 7998.

18 Y.-X. Du, H.-T. Sheng, D. Astruc and M.-Z. Zhu, Atomically precise noble metal nanoclusters as efficient catalysts: a bridge between structure and properties, Chem. Rev., 2020, 120,526 .

19 T.-Y. Chen, B.-T. Chen, K. V. Bukhryakov and V. O. Rodonov Thiols make for better catalysts: Au nanoparticles supported on functional SBA-15 for catalysis of Ullmann-type homocouplings, Chem. Commun., 2017, 53, 11638.

20 W. Yao, W.-J. Gong, H.-X. Li, F.-L. Li, J. Gao and J.-P. Lang, Synthesis of DMF-protected Au NPs with different size distributions and their catalytic performance in the Ullmann homocoupling of aryl iodides, Dalton Trans., 2014, 43, 15752.

21 K. Layek, H. Maheswaran and M. L. Kantam, Ullmann coupling of aryl iodides catalyzed by gold nanoparticles stabilized on nanocrystalline magnesium oxide, Catal. Sci. Technol., 2013, 3, 1147.

22 M. Dabiri, M. Shariatipour, S. K. Movahed and S. Bashiribod, Water-dispersible and magnetically separable gold nanoparticles supported on a magnetite/s-graphene nanocomposite and their catalytic application in the Ullmann coupling of aryl iodides in aqueous media, RSC Adv., 2014, 4, 39428.

23 S. K.Movahed, M. Fakharian, M. Dabiri and A. Bazgir, Gold nanoparticle decorated reduced graphene oxide sheets with high catalytic activity for Ullmann homocoupling, RSC Adv., 2014, 4, 5243.

24 D. Minoo, K. S. R. Banifatemi, L. N. Farajinia and B. Sahareh, Synthesis of gold nanoparticles decorated on sulfonated three-dimensional graphene nanocomposite and application as a highly efficient and recyclable heterogeneous catalyst for Ullmann homocoupling of aryl iodides and reduction of $p$ nitrophenol, Appl. Organometal. Chem., 2018, 32, e4189.

25 J. Jiang, L.-Y. Du and Y.-Q. Ding, Aryl-aryl bond formation by Ullmann reaction: from mechanistic aspects to catalyst, Minirev. Org. Chem., 2020, 17, 26.

26 A. Dhackshinamoorthy, A. M. Asiric and H. Garcia, Chem. Soc. Rev., 2015, 44, 1922.

27 Q. Wang and D. Astruc, State of the art and prospects in metal-organic framework (MOF)-based and MOF-derived nanocatalysis, Chem. Rev., 2020, 120, 1438.

$28 \mathrm{M}$. T. Reetz and J. G. de Vries, Ligand-free Heck reactions using low Pd-loading, Chem. Commun., 2004, 14, 1559.

29 D. Astruc, F. Lu and J. Ruiz, Nanoparticles as recyclable catalysts: the frontier between homogeneous and heterogeneous catalysis, Angew. Chem., Int. Ed., 2005, 44, 7852.

30 R. W. J. Scott, O.M. Wilson and R. M. Crooks, Synthesis, characterization, and applications of dendrimerencapsulated nanoparticles, J. Phys. Chem. B., 2005, 109, 692
31 D. Astruc, Palladium catalysis using dendrimers: molecular catalysts versus nanoparticles, Tetrahedron Asymm., 2010, 21, 1041.

32 L. M. Bronstein and Z. B. Shifrina, Dendrimers as encapsulating, stabilizing, or directing agents for inorganic nanoparticles, Chem. Rev., 2011, 111, 5301.

33 K. Philippot and P. Serp, Concepts in nanocatalysis, Nanomaterials in Catalysis (Eds. P. Serp and K. Philippot), Wiley-VCH, Weinheim, 2013.

34 D. Astruc, Introduction: nanoparticles in catalysis, Chem. Rev., 2020, 120, 461

35 M. K. Samantaray, V. D'Elia, E. Pump, L. Falivene, M. Harb S. O. Chikh, L. Cavallo and J.-M. Basset, The comparison between single atom catalysis and surface organometallic catalysis, Chem. Rev., 2020, 120, 734.

36 A. R. Tao, S. Habas and P.-D. Yang, Shape control of colloidal metal nanocrystals, Small, 2008, 4, 310.

37 N. T. S. Phan, M. van der Sluys and C. W. Jones, On the nature of the active species in palladium catalyzed MizorokiHeck and Suzuki-Miyaura couplings-homogeneous or heterogeneous catalysis, a critical review, Adv. Syn. Catal. 2006, 348, 609.

38 F.-Y. Fu, C.-L. Wang, Q. Wang, A. M. Martinez-Villacorta, A Escobar, H. B. Chong, X. Wang, S. Moya, L. Salmon, E. Fouquet, J. Ruiz and D. Astruc, Highly selective and sharp volcano-type synergistic $\mathrm{Ni}_{2} \mathrm{Pt} @ \mathrm{ZIF}$-8-catalyzed hydrogen evolution from ammonia borane hydrolysis, J. Am. Chem. Soc., 2018, 140, 10034.

39 C.-L. Wang, J. Tuninetti, Z. Wang, C. Zhang, R. Ciganda, L. Salmon, S. Moya, J. Ruiz and D. Astruc, Hydrolysis of ammonia-borane over Ni/ZIF-8 nanocatalyst: high efficiency, mechanism, and controlled hydrogen release, J. Am. Chem. Soc., 2017, 139, 11610.

40 S.-N. Zhao, X.-Z. Song, S.-Y. Song and H.-J. Zhang, Highly efficient heterogeneous catalytic materials derived from metal-organic framework supports/precursors, Coord. Chem. Rev., 2017, 337, 80.

41 K. Shen, X.-D. Chen, J.-Y. Chen and Y.-W. Li, Development of MOF-derived carbon-based nanomaterials for efficient catalysis, ACS Catal., 2016, 6, 5887.

42 K. S. Park, Z. Ni, A. P. Cote, J. Y. Choi, R. Huang, F. J. UribeRomo, H. K. Chae, M. O'Keeffe and O. M. Yaghi, Exceptional chemical and thermal stability of zeolitic imidazolate frameworks, Proc. Natl. Acad. Sci. U. S. A., 2006, 103, 10186

43 J. Cravillon, R. Nayuk, S. Springer, A. Feldhoff, K. Huber and M. Wiebcke, Controlling zeolitic imidazolate framework nano-and microcrystal formation: insight into crystal growth by time-resolved in situ static light scattering, Chem. Mater., 2011, 23, 2130

44 M.-T. Zhao, K. Yuan, Y. Wang, G.-D. Li, J. Guo, L. Gu, W.-P. Hu, H.-J. Zhao and Z.-Y. Tang, Metal-organic frameworks as selectivity regulators for hydrogenation reactions, Nature, 2016, 539, 76.

45 D. Astruc. Organometallic Chemistry and Catalysis. Springer, Heidelberg, 2007, Chapter 15.

46 M. Yurderi, A. Bulut, M. Zahmakiran, M. Gülcan and S. Özkar, Ruthenium(0) nanoparticles stabilized by metal-organic framework (ZIF-8): Highly efficient catalyst for the dehydrogenation of dimethylamine-borane and transfer hydrogenation of unsaturated hydrocarbons using dimethylamine-borane as hydrogen source, Appl. Catal. BEnviron., 2014, 160, 534.

47 J. K. Kochi. Organometallic Mechanisms and Catalysis, Academic Press, New York, 1978, Chap. 7.

48 D. Astruc. Electron Transfer and Radical Processes in Transition Metal Chemistry, VCH, New York, 1995, Chap.1. 
49 I. Pastoriza-Santos and L. M. Liz-Marzan, N,NDimethylformamide as a reaction medium for metal nanoparticle synthesis, Adv. Funct. Mater., 2009, 19, 679.

50 A. Loupy, B. Tchoubar, D. Astruc, Salt effects resulting from exchange between two ion pairs and their crucial role in reaction, Chem. Rev., 1992, 92, 1141.

51 A. Loupy, B. Tchoubar. Salt Effects in Organic and Organometallic Chemistry, VCH, Weinheim, 1991.

\section{Table of content}

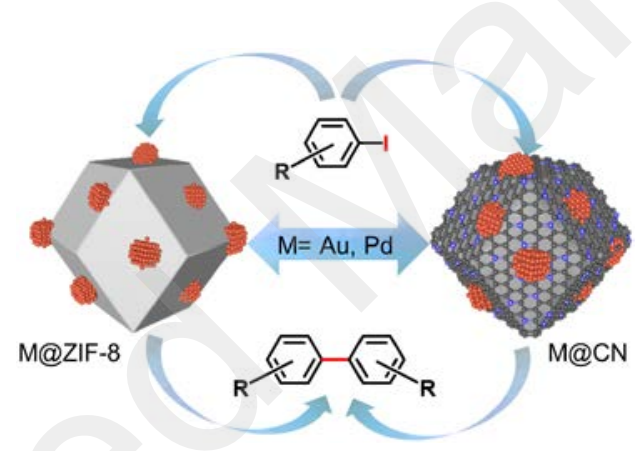

Compared ZIF-8-based and ZIF-8-derived gold and palladium nanocatalysts are very efficient for optimized Ullmann coupling of iodoarenes in DMF. 